\title{
Data-Driven Iterative Vibration Signal Enhancement Strategy Using Alpha Stable Distribution
}

\author{
Grzegorz Żak, ${ }^{1}$ Agnieszka Wyłomańska, ${ }^{2}$ and Radosław Zimroz ${ }^{1}$ \\ ${ }^{1}$ Diagnostics and Vibro-Acoustics Science Laboratory, Wrocław University of Science and Technology, Na Grobli 15, \\ 50-421 Wrocław, Poland \\ ${ }^{2}$ KGHM Cuprum Research \& Development Center, Ul. Sikorskiego 2-8, 53-659 Wrocław, Poland \\ Correspondence should be addressed to Grzegorz Żak; grzegorz.zak@pwr.edu.pl
}

Received 12 June 2017; Accepted 26 July 2017; Published 12 September 2017

Academic Editor: Andrzej Katunin

Copyright (C) 2017 Grzegorz Żak et al. This is an open access article distributed under the Creative Commons Attribution License, which permits unrestricted use, distribution, and reproduction in any medium, provided the original work is properly cited.

\begin{abstract}
The authors propose a novel procedure for enhancement of the signal to noise ratio in vibration data acquired from machines working in mining industry environment. Proposed method allows performing data-driven reduction of the deterministic, high energy, and low frequency components. Furthermore, it provides a way to enhance signal of interest. Procedure incorporates application of the time-frequency decomposition, $\alpha$-stable distribution based signal modeling, and stability parameter in the time domain as a stoppage criterion for iterative part of the procedure. An advantage of the proposed algorithm is data-driven, automative detection of the informative frequency band as well as band with high energy due to the properties of the used distribution. Furthermore, there is no need to have knowledge regarding kinematics, speed, and so on. The proposed algorithm is applied towards real data acquired from the belt conveyor pulley drive's gearbox.
\end{abstract}

\section{Introduction}

Local damage detection in rotating machines is one of the most frequent topics in condition monitoring literature. Generation of such signal is well recognized ([1-4]). Basically, the problem is related to detection of cyclic impulsive disturbances in noisy observation. Different approaches have been used for signal modeling (cyclostationary [5], stochastic [6], autoregressive [7]), enhancement (SOI extraction [810], denoising including adaptive noise cancellation [11] and spatial wavelet denoising [12], averaging: time domain [13, 14], time-frequency domain), sources separation (discreterandom [15]), damage detection criteria (kurtosis, cyclostationary indicators, and statistical measures) $[4,10,16-18]$, and so on. Special attention was paid to damage detection at early stage of development, in nonstationary speed/load condition, and so on. In the literature one can find several interesting reviews concerning mentioned problem to get holistic, more detailed view [19-22].

One might conclude that almost everything was done to be able to diagnose damage. However, in the practical applications there are plenty of challenging cases that prove difficult for the classical methods. In this paper we will present an interesting case related to heavy duty gearbox operating in harsh environment. Based on this example, we propose novel, data-driven procedure for damage detection. An important fact is that there are two damage types (with different nature and localization). One of them is easy to notice directly from raw signal. However, the second one produces weak signature and is hardly detectable. We have started with most popular tools as spectral kurtosis-based filter and envelope analysis. Unfortunately, the results are not satisfactory. So it motivates us to search for alternative solutions. As mentioned, it is expected that signal of interest (SOI) will be impulsive. There are plenty of techniques in time series analysis that are focused on data with such behavior. One can easily notice increasing number of publications concerning application of heavy-tailed distributions towards vibration and sound signals [23-27]. Such interest is especially aimed towards $\alpha$-stable distribution which is a generalization of Gaussian one. In [23] one can find a thorough description on how to model and apply such distribution towards data. Modifications and extensions of existing methods towards 
heavy-tailed distribution can be found in the literature with promising results ([24-27]).

It motivates us to test our recently developed tools related for $\alpha$-stable distribution based filtering procedure. Again it was slightly better (improvement noticeable at spectrogram) but still requires advanced interpretation. We have identified that the problem is related to signal structure and high energy concentration in low frequency range of spectrum. Filtering is just simply multiplication of complex spectrum of signal with filter characteristics in frequency domain. Even small values of filter coefficients at low frequencies might result in still poor signal to noise ratio (SNR) in output signal. Our strategy is instead of direct extraction of SOI, we propose attenuating noninformative high energy signal components and then use $\alpha$-stable distribution based filter to extract SOI. Such strategy appeared to be very effective as presented in [28]. A key question is how to design filter to attenuate noninformative part. We propose using $\alpha$-stable distribution approach (we already use it for SOI extraction based on $\alpha$ parameter); however, in first stage we will use scale parameter $\sigma$. We mention that the $\alpha$ parameter gives information on how heavy the distribution tail is and $\sigma$ is responsible for the scale of the distribution. During experiments it has appeared that $\sigma$-based filtering performed several times provides much better results than just one filtration. Obviously, we immediately tested iterative $\alpha$-based filtering for SOI extraction but without spectacular success. Finally, we propose a two-stage procedure for signal preprocessing:

(i) Iterative $\sigma$-based filtering with stability parameter in the time domain as stoppage criterion for high energy noninformative part attenuation.

(ii) $\alpha$-based filtering for further SOI signal enhancement.

In the end, this paper combines method of data-driven filtering together with heavy-tailed distribution modeling. Such connection provides new insight towards modeling of subsignals from time-frequency decomposed signal.

The paper is organized as follows: Section 1 contains introduction about the topic of paper together with short summary of work done in the field. In Section 2 we present methodology associated with our procedure and in Section 3 we apply our procedure towards real vibration data from the gearbox of drive pulley of belt conveyor. In Section 4 we summarize results and provide conclusions.

\section{Methodology}

In this section we present the methodology useful in the problem of local damage detection based on the analysis of the vibration signal. We propose the approach based on the analysis of subsignals obtained in time-frequency representation (spectrogram) of given signal. Mentioned subsignals are analyzed using appropriate statistics (called selectors). Till now, the most popular statistic was kurtosis, one of the measures that can point out these frequency bins on timefrequency map that reveals the most impulsive nature. When the kurtosis is applied to the appropriate subsignals, then it is called the spectral kurtosis (SK), [10]. We recall that the spectrogram $\operatorname{spec}(t, f)$ for given time point $t \in T$ and frequency $f \in F$ is defined through the short time Fourier transform (STFT) in the following way [29]:

$$
\operatorname{spec}(t, f)=|\operatorname{STFT}(t, f)|^{2}=\sum_{k=0}^{n-1} x_{k} w(t-k) e^{2 j \pi f k / n},
$$

where $w(t-k)$ is the shifting window and $x_{k}$ is the input signal $(k=0,1, \ldots, n-1)$. Parameters for the spectrogram should be chosen with user preferences for best visibility of the impulsive behavior in the visual representation of the spectrogram. Parameters to be chosen are window length, number of samples overlapping, and number of samples used for fast Fourier transform (nfft). In case of the vibration signals acquired from pulley drive gearbox for belt conveyor analyzed in Section 3 we found them to be optimal at the following levels: window length equal to 256 samples, number of samples overlapping equal to 240 samples, and number of samples used for the FFT equal to 512 samples.

Thus the spectral kurtosis (SK) statistic for input signal $x_{k}(k=0,1, \ldots, n-1)$ is defined in the following way [10]:

$$
\operatorname{SK}(f)=\# T \frac{\sum_{t \in T}|\operatorname{STFT}(t, f)|^{4}}{\left(\sum_{t \in T}|\operatorname{STFT}(t, f)|^{2}\right)^{2}}-2,
$$

where \#T denotes the number of elements of the set $T$, that is, number of time points at which STFT is calculated.

However for some real signals the spectral kurtosis does not give expected results because it can be sensitive for impulses not related to damage (i.e., artifacts). Therefore, as it was mentioned, there are other statistics considered that can be applied instead of the kurtosis; see [16].

In this paper we propose not to calculate simple statistic for set of subsignals obtained by decomposition of raw data by spectrogram but to describe each subsignal by stochastic model that has similar properties as appropriate time series. One of the easiest stochastic models is based on the assumption that the vector of observations contains realizations of independent identically distributed random variables. The most known distribution is the Gaussian one. However, the Gaussian distribution is not appropriate to modeling data with impulses, like for instance subsignals from timefrequency representation (spectrogram) related to damage. It is more convenient to take under consideration more general distribution, that is, such that it can be appropriate to describe subsignals corresponding to informative frequency band (IFB) and from noninformative frequency bands. Of course for those regions the parameters of the chosen distribution will be different. One of the possibilities is the $\alpha$-stable distribution [30], which is a generalization of the Gaussian one. The random variable $X$ has $\alpha$-stable distribution if its characteristic function is given by

$$
\begin{aligned}
& \phi_{X}(t)=E \exp (i t X) \\
& = \begin{cases}\exp \left\{-\sigma^{\alpha}|t|^{\alpha}\left(1-i \beta \operatorname{sign}(t) \tan \left(\frac{\pi \alpha}{2}\right)\right)+i \mu t\right\}, & \alpha \neq 1, \\
\exp \left\{-\sigma|t|\left(1+i \beta \frac{2}{\pi} \operatorname{sign}(t) \ln (|t|)\right)+i \mu t\right\}, & \alpha=1 .\end{cases}
\end{aligned}
$$




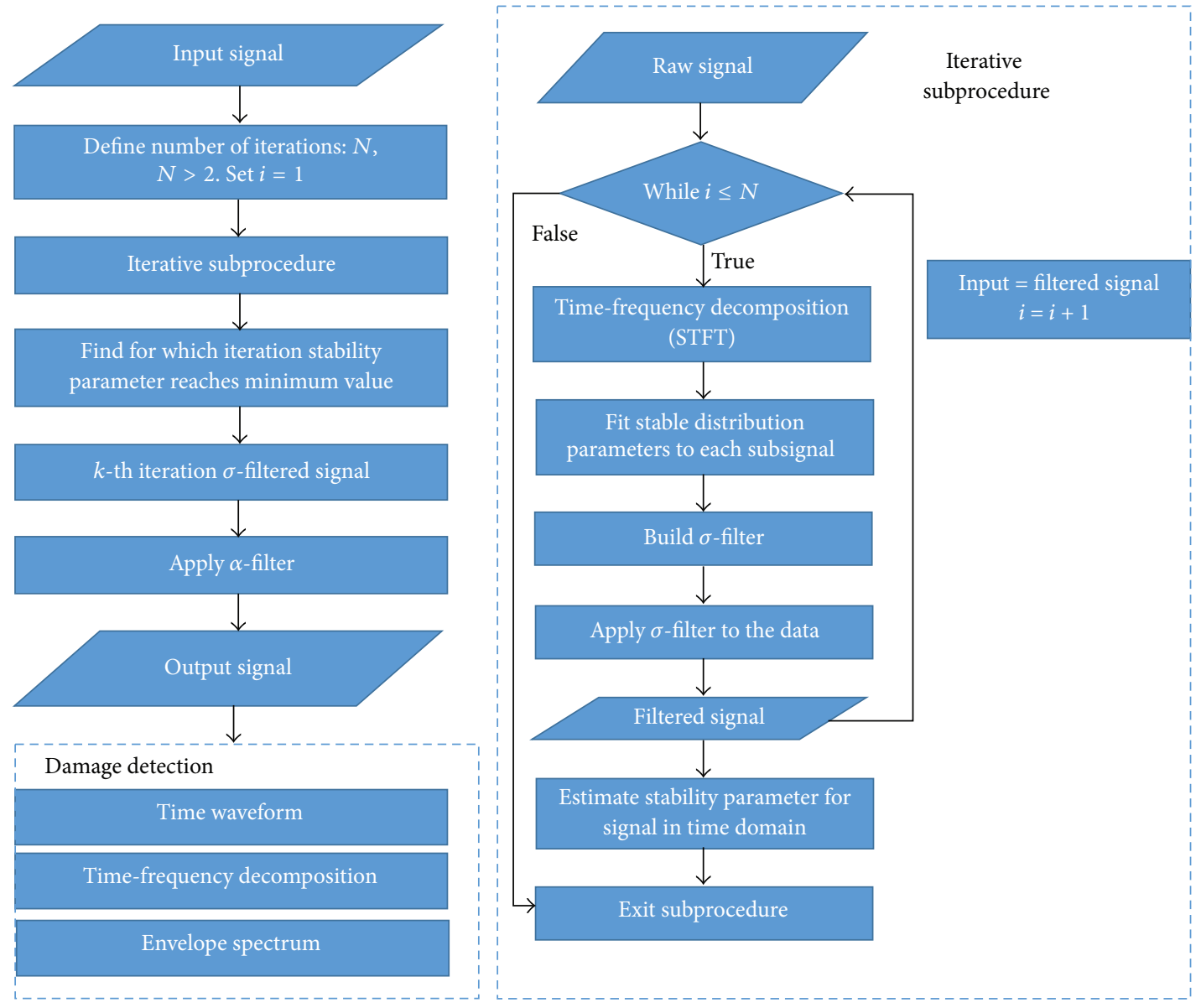

FIGURE 1: Flowchart of the procedure.

We provide short description of distribution parameters. Stability parameter $\alpha \in(0,2]$ defines how impulsive the realizations of the distribution are. There is a relation between $\alpha$ value and impulsiveness of the signal, with lower values we observe more impulsive behavior. The parameter $\sigma>0$ is responsible for the scale (or energy) of the distribution. It behaves in a similar way to the variance of the Gaussian case (i.e., for $\alpha=2$ ). The parameter $\beta \in[-1,1]$ is responsible for the distribution skewness and $\mu \in \mathbb{R}$ represents distribution shift. It corresponds to the mean for $1<\alpha \leq 2$ and the median for $0<\alpha \leq 1$. It is worth mentioning that, in general, the probability density function, as well as the cumulative distribution function for $\alpha$-stable distributed random variable, is not given in explicit form. There are only three exceptions: Gaussian, Levy, and Cauchy distributions. Moreover, finite $p$ th moments exist only for $p<\alpha$. The $\alpha$-stable distribution and processes have found many interesting applications, also in technical diagnostics [24]. In our approach, instead of kurtosis calculation for each subsignal from time-frequency representation, we propose examining the $\alpha$ and $\sigma$ parameters calculated on the basis of appropriate subsignals. We estimate those parameters by using the regression method [31], where it is widely discussed. Whole idea is based on the regression of the characteristic function of the sample.
Here, we use parameters $\alpha$ and $\sigma$ as indicators of impulsivity and energy, respectively.

Subsignals coming from bands with high energy should have significantly higher scale parameter. High energy in the spectrogram of the vibration signal from gearbox is connected with the deterministic component of the signal. Combining such information one can construct filter characteristic which will allow for deterministic component attenuation. Filter construction is as follows. Let us assume that estimated parameters $\sigma$ of $\alpha$-stable distribution for set of subsignals extracted from the time-frequency decomposition are denoted as $\widehat{\sigma}=\left\{\widehat{\sigma}\left(f_{1}\right), \widehat{\sigma}\left(f_{2}\right), \ldots, \widehat{\sigma}\left(f_{M}\right)\right\}$, where $M=$ $\lfloor\mathrm{nfft} / 2\rfloor+1$. We define filter characteristic as follows:

$$
\sigma_{\text {filter }}(f)=1-\frac{\widehat{\sigma}(f)}{\max (\widehat{\sigma})} .
$$

We define stoppage criterion as minimum value of the stability parameter of the signal in the time domain. This parameter indicates impulsivity of the data. The lower the value of this parameter, the higher the impulsivity. One of the other approaches would be to use kurtosis as indicator. However, it is not suggested as it can be easily affected by single impulses that are not related to the fault.

Filtered signal is now assumed to be input signal for the spectrogram and whole procedure restarts at calculation of 


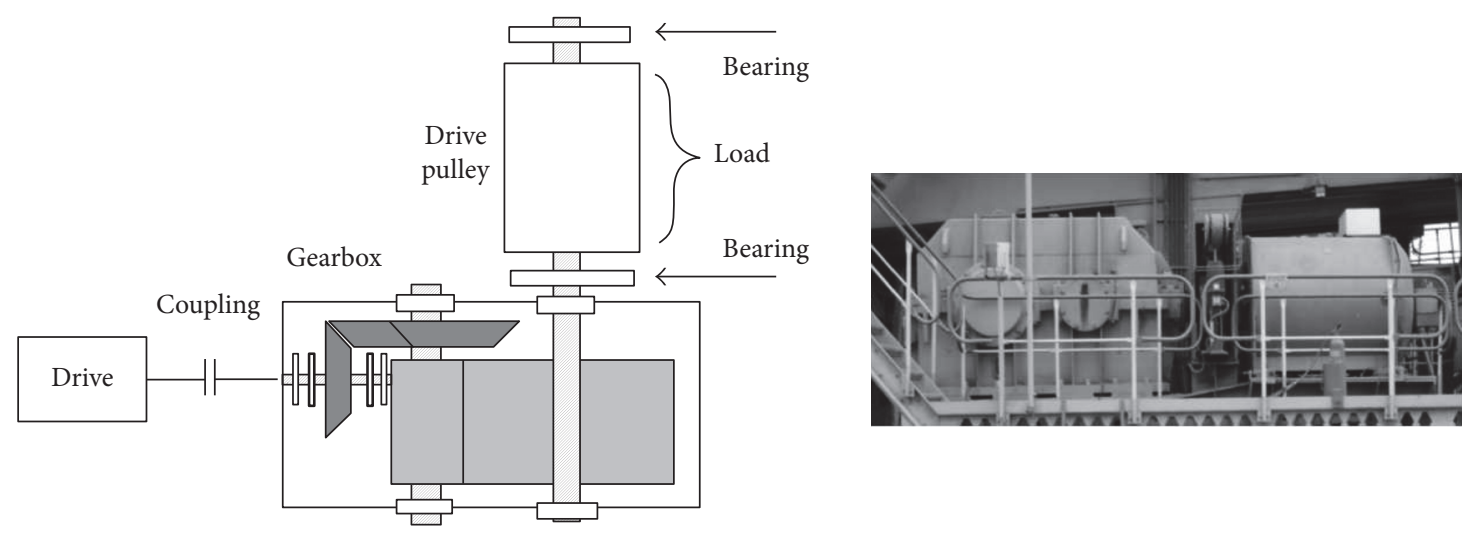

FIGURE 2: Investigated machine.

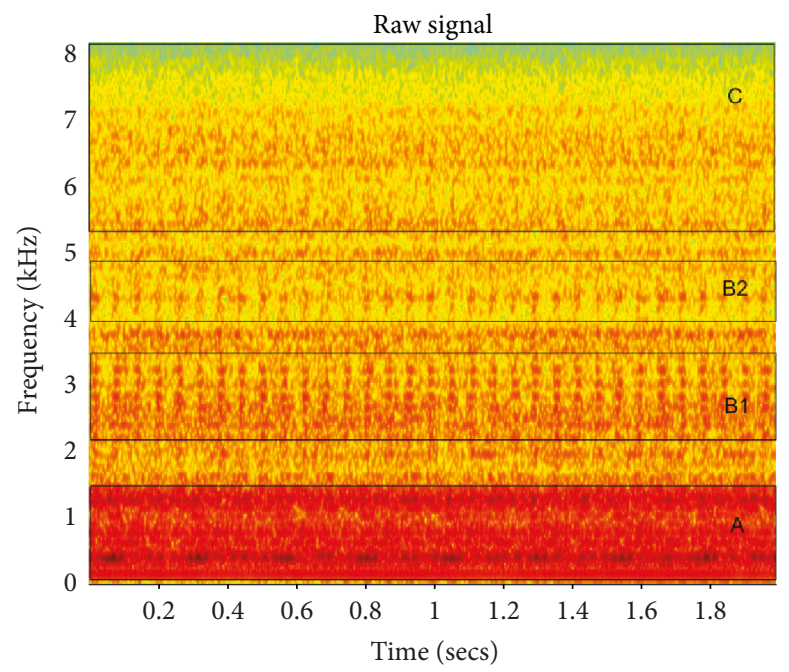

(a)

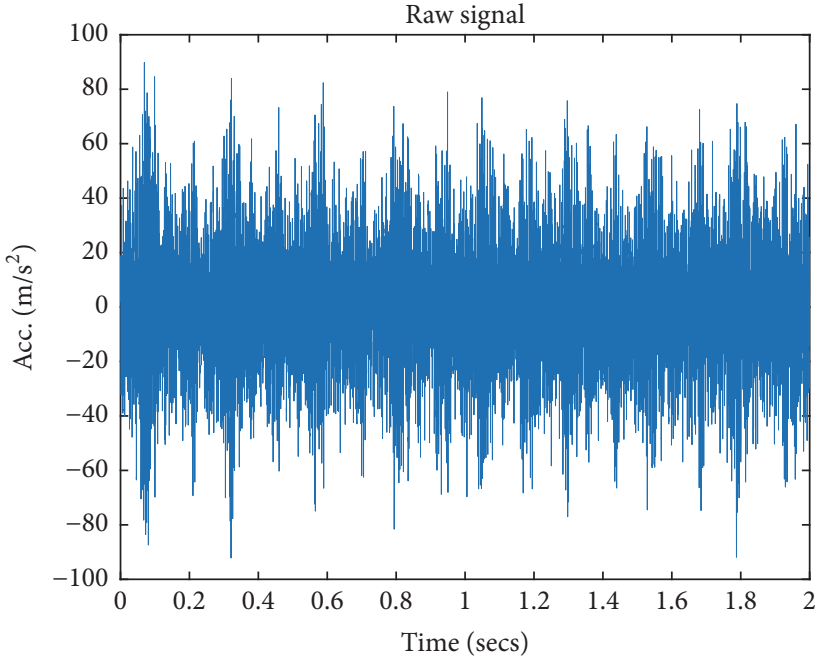

(b)

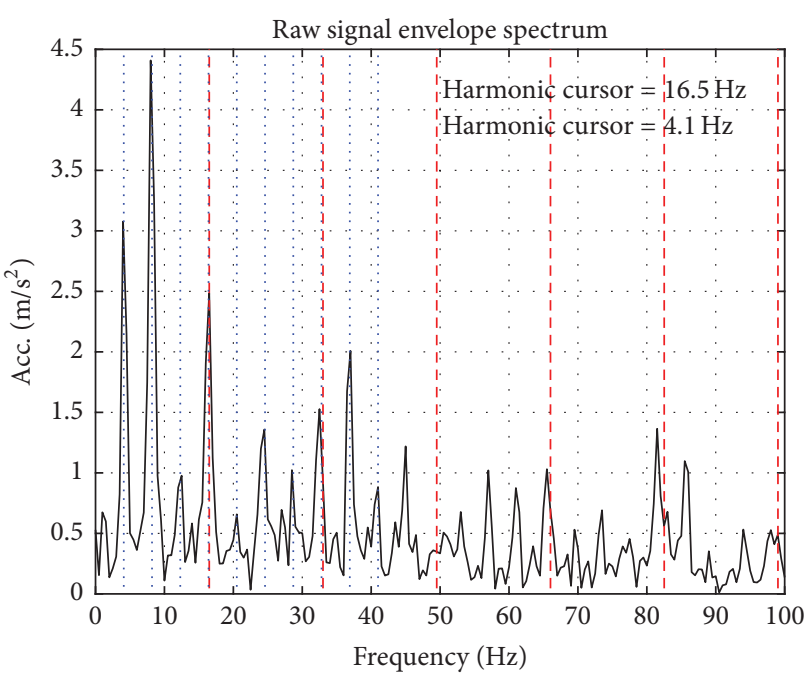

(c)

FIGURE 3: Spectrogram of the signal (a), time waveform of the signal (b), and its envelope spectrum (c). 


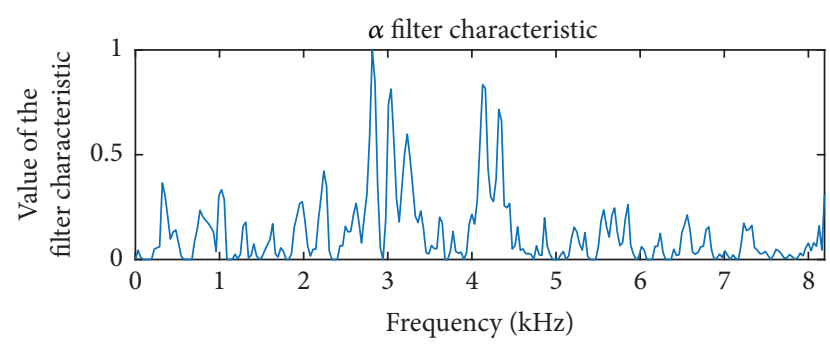

(a)

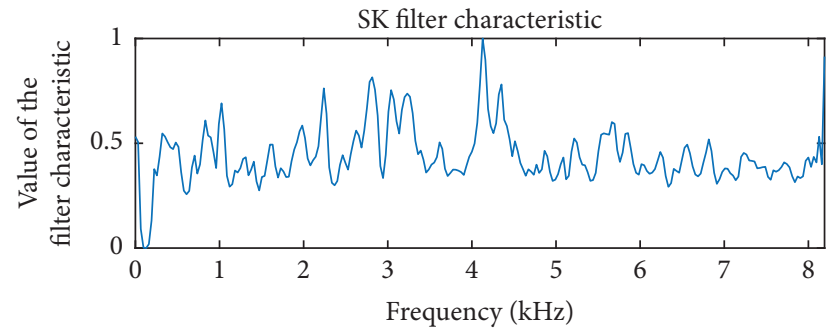

(b)

FIGURE 4: Filter characteristics based on the $\alpha$-stable distribution (a) and spectral kurtosis (b).

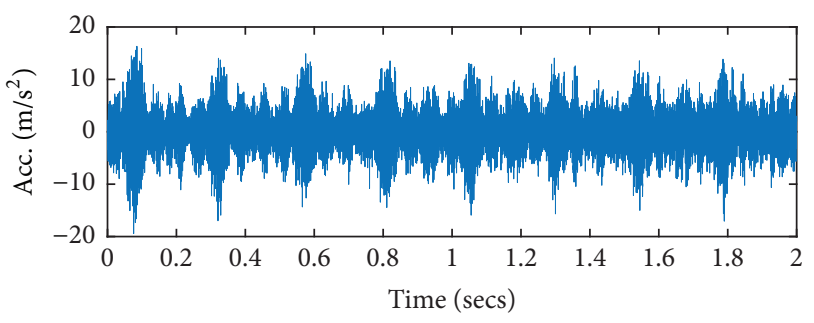

(a)

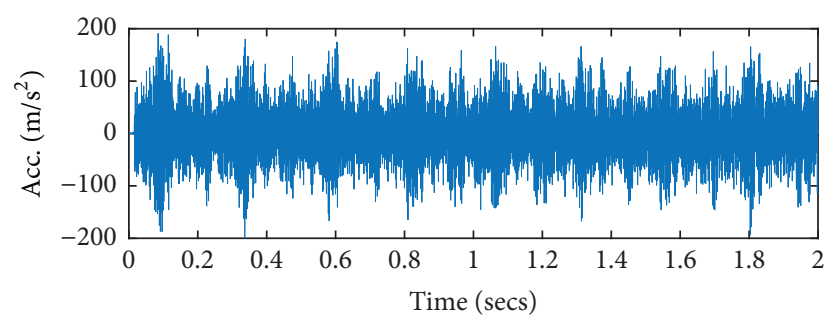

(b)

FIGURE 5: Time waveforms of $\alpha$-filtered signal (a) and SK-filtered signal (b).

the spectrogram with repeated filtrations until $N$ iterations. After performing all iterations one needs to find in which iteration the stability parameter of the signal in time domain reaches the minimum value for the filtered signal. This allows us to determine where deterministic component has been attenuated best. Filtered signal from that iteration has now highly attenuated deterministic component. In addition, we can use $\alpha$-filtration [24] to easily enhance impulsive component of the signal. Let us assume that estimated vector of $\alpha$ parameters for subsignals from time-frequency

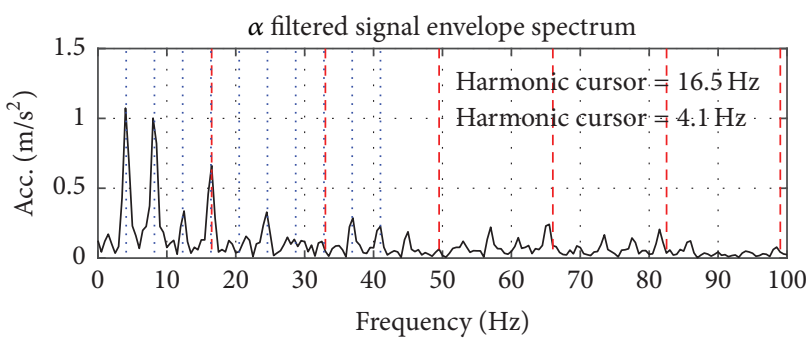

(a)

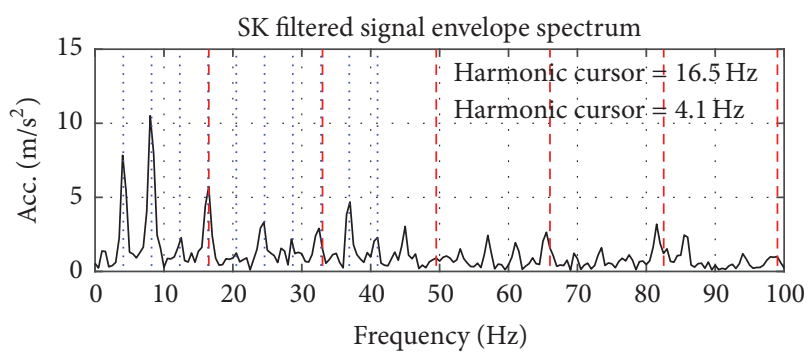

(b)

FigURE 6: Envelope spectra of $\alpha$-filtered signal (a) and SK-filtered signal (b).

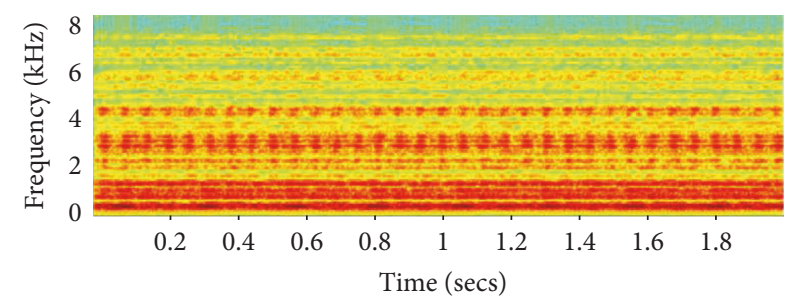

(a)

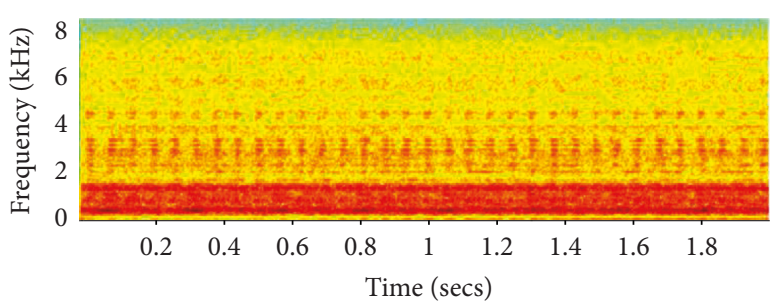

(b)

FIGURE 7: Spectrograms of $\alpha$-filtered signal (a) and SK-filtered signal (b).

representation is given by $\widehat{\alpha}=\left\{\widehat{\alpha}\left(f_{1}\right), \widehat{\alpha}\left(f_{2}\right), \ldots, \widehat{\alpha}\left(f_{M}\right)\right\}$. Then $\alpha$ filter characteristic would be defined as

$$
\alpha_{\text {filter }}(f)=\frac{\max (\widehat{\alpha}(f))-\widehat{\alpha}(f)}{\max (\widehat{\alpha}(f))-\min (\widehat{\alpha}(f))} .
$$

Output signal has now significantly reduced deterministic component, enhanced impulsive component, and can be easily analyzed in time, frequency, and time-frequency domains. In Figure 1 we present the flowchart of the described procedure. 


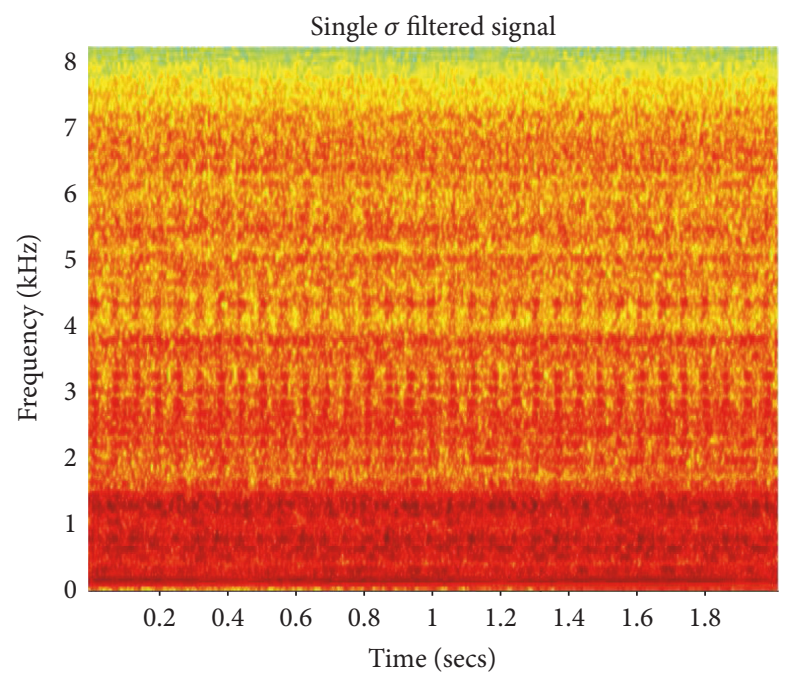

(a)

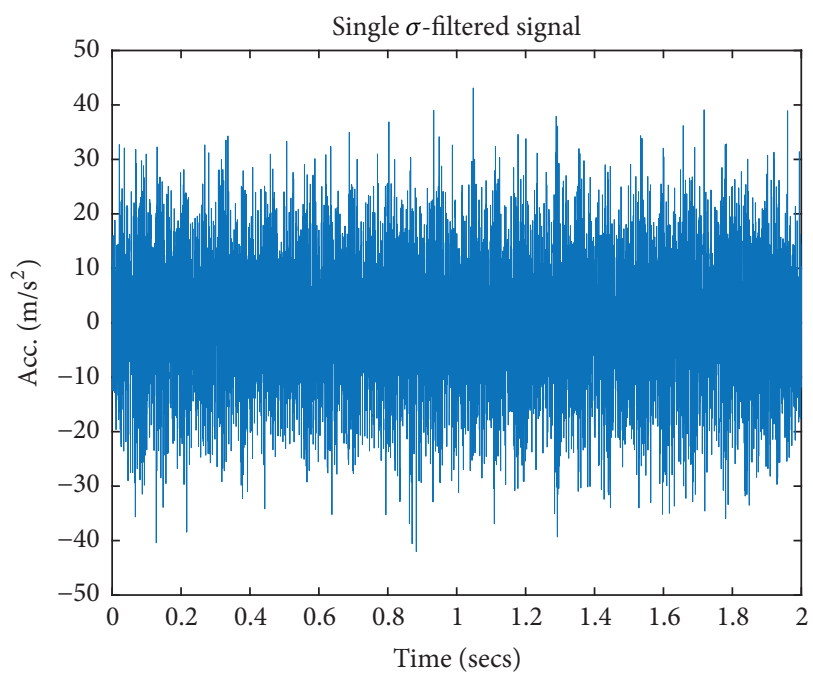

(b)

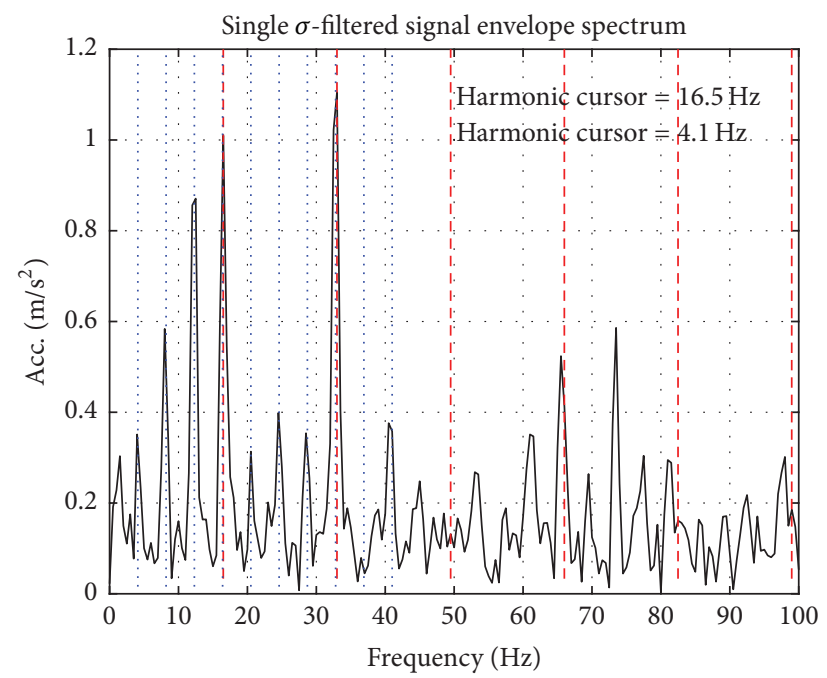

(c)

FIGURE 8: Spectrogram of the signal (a), time waveform of the signal (b), and its envelope spectrum (c).

\section{Real Data Analysis}

To prove efficiency of the proposed methodology we will show results of application of data-driven filtration to real vibration data from complex mechanical system operating in mining industry (Figure 2). Moreover, gearbox comes from driving system used in belt conveyor, a very popular technology, commonly used in mining industry for material transportation. Measurements have been performed using Bruel Kjaer Pulse system. Parameters of data acquisition depend on the investigated object. In this case, they are as follows: duration $2 \mathrm{~s}$, sampling frequency $16384 \mathrm{~Hz}$, and the expected faults frequencies are $4.1 \mathrm{~Hz}$ and $16.5 \mathrm{~Hz}$. This section is divided into two subsections. First subsection contains results of applying spectral kurtosis method for enhancement of the impulsive component in the signal and applying $\alpha$ filter. Second subsection contains novel procedure, namely, data-driven filtration described in Section 2.
In Figure 3 one can observe time waveform of the signal, its envelope spectrum, and its time-frequency decomposition, namely, spectrogram. There are presently 3 main type frequency bands in the spectrogram: first containing low frequency high energy component responsible for the shape of the signal (marked with A), second being informative frequency bands (IFB) placed at $2.5-3.5 \mathrm{kHz}$ and $4-4.5 \mathrm{kHz}$ (marked with $\mathrm{B} 1$ and $\mathrm{B} 2$, resp.), and noninformative high frequency band (marked with C). Furthermore, it can be easily seen that it is correct placement of IFBs when observing values of $\alpha$-stable distribution parameter estimated for each frequency bin (Figure 4(a)). In Figure 3(c) one can see envelope spectrum of the signal with marked frequencies of both faults with its harmonics. First fault with frequency equal to $4.1 \mathrm{~Hz}$ (blue vertical lines) overlaps second fault with frequency equal to $16.5 \mathrm{~Hz}$ (red vertical lines). Due to the operation regime of the machine it was not possible to perform visual inspection. Parameters of the machine are 

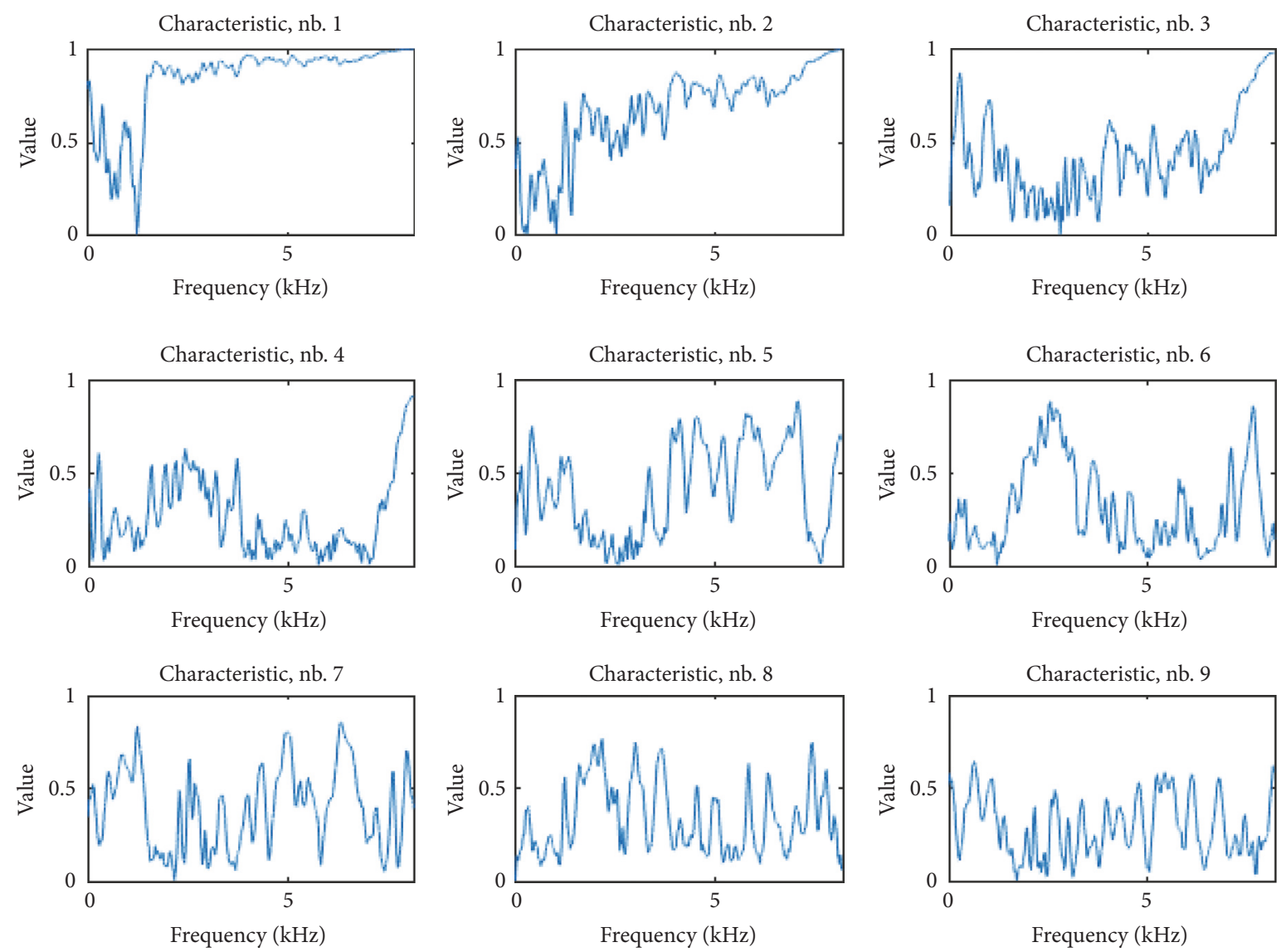

FIGURE 9: $\sigma$-filter characteristics for each iteration.

known including shaft and mesh characteristic frequencies. It allows for the rough fault detection in time domain. However, second fault is not visible in any domain.

3.1. Spectral Kurtosis and $\alpha$ Filtration Comparison. This subsection contains results of application of spectral kurtosis to the vibration data measured on the gearbox casing and moreover it contains authors' procedure of $\alpha$-stable distribution based filtration. Both spectral kurtosis and $\alpha$ act in the literature as measures of impulsivity of the subsignals from the spectrogram.

In Figure 4 one can observe filter characteristics for filtration methods based on the $\alpha$-stable distribution (a) and spectral kurtosis (b). One can denote more precise informative band selection in the method using $\alpha$-stable distribution.

Using obtained filter characteristics one can perform filtration of the raw signal. The following figures contain filtered signals in different domains. In Figure 5 one can observe result of the filtration in the time domain. In comparison to the raw signal time waveform it can be denoted that impulsive component has been slightly enhanced.

However, comparing envelope spectra (Figure 6) one cannot easily determine existence of both faults in the frequency domain due to the visible fault overlapping hidden fault. Such situation happened due to the second fault frequency being

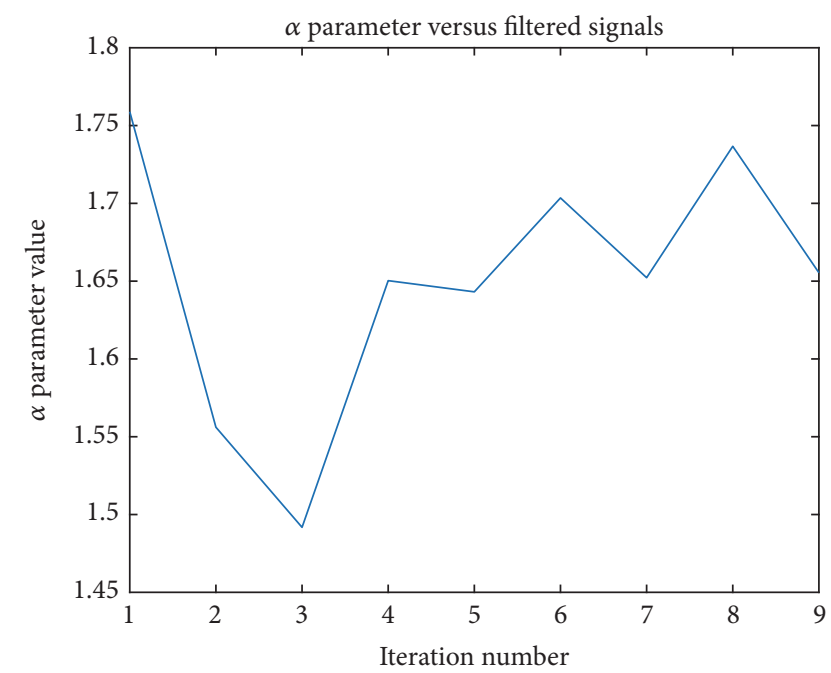

FIGURE 10: Stability parameter for each $\sigma$-filtered signal.

multiplicative of the first one, the same as in unprocessed signal envelope spectrum.

It can be seen here in Figure 7 that $\alpha$ filter heavily enhanced impulsive component in its bands while SK results are poor in this case. 


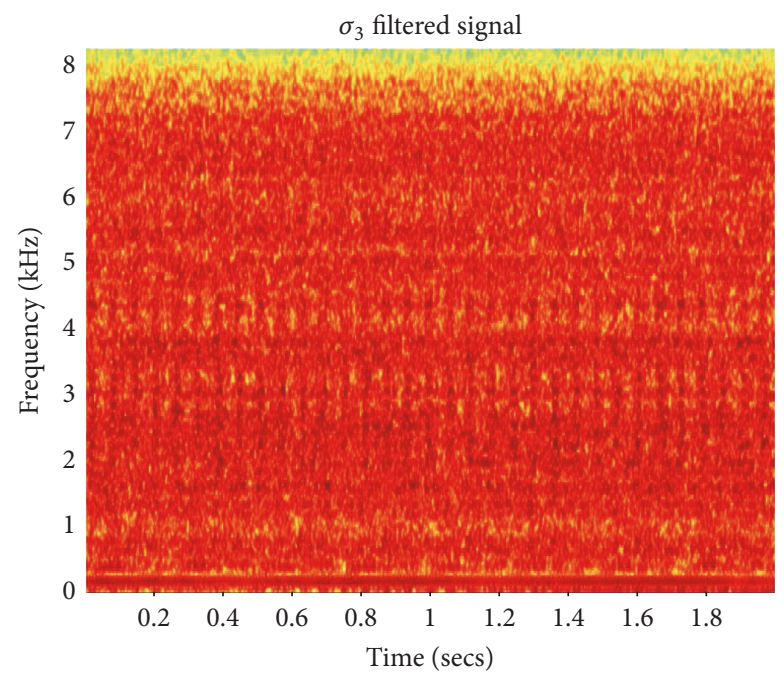

(a)

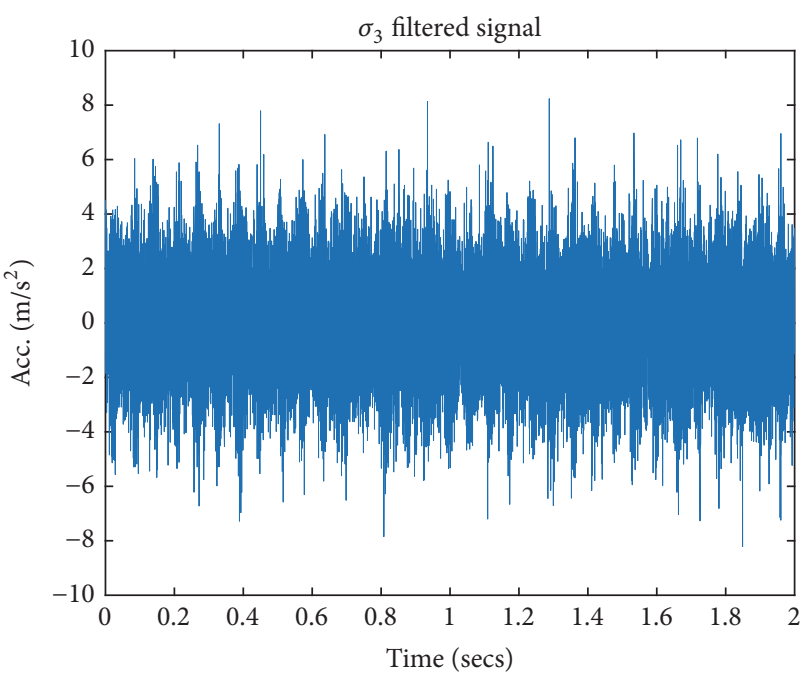

(b)

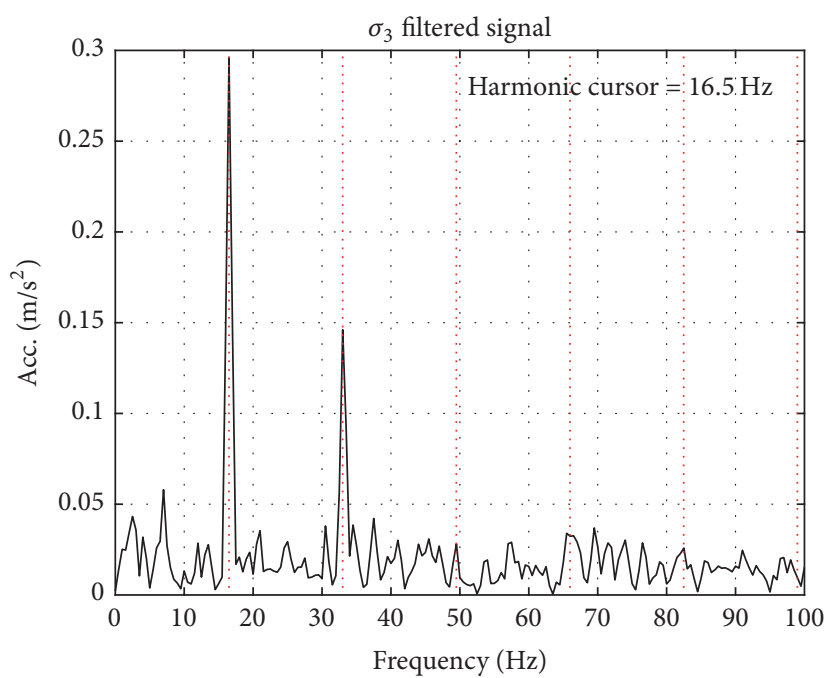

(c)

FIGURE 11: Spectrogram of the signal (a), time waveform of the signal (b), and its envelope spectrum (c).

Further iterations of $\alpha$-filter do not improve SNR due to the behavior of the model. Fitting $\alpha$-stable distribution to the signal filtered with $\alpha$-filter will result in similar distribution of the $\alpha$ parameters.

3.2. Novel Method Results. In this subsection one can denote that it contains results for the single $\sigma$-filtration which will provide reference point together with $\alpha$-filtration described in previous section, consecutive filter characteristics for each of the iterative $\sigma$-filtrations, and finally result of applying $\alpha$-filter to the $\sigma$-filtered signal with minimum value of the stability parameter for the signals in the time domain.

As for the comparison to the $\alpha$-filtration we performed $\sigma$-filtration by fitting $\alpha$-stable distribution towards subsignals extracted from the time-frequency decomposition of the raw vibration signal. Combining information visible in Figure 8 one can see that deterministic component of the signal has been attenuated (Figures 8(a) and 8(c)) providing even distribution of the energy on the spectrogram. Furthermore, envelope spectrum allows one to see fundamental frequency of the hidden fault along with its harmonics. However, time waveform does not yet provide important result about the fault.

Based on results from $\alpha$-filtration (Figures 5-7) and $\sigma$ filtration (Figure 8) we can say that neither $\alpha$ nor $\sigma$ filtration provide satisfactory results. Both improve slightly SNR but results are not perfect. Given properties of both parameters one can apply procedure from Figure 1 iteratively. Given raw vibration signal it is necessary to decompose it via STFT. Further, one establishes $\sigma$-filter characteristics (Figure 9) and filters signal and the procedure is repeated.

Each of the filtrations is based on the $\alpha$-stable distribution and its scale parameter, namely, $\sigma$. Filtering iteratively demands fitting mentioned distribution to the subsignals extracted from the time-frequency decomposed signal and further filtered signals. It allows for the creation of the $\sigma$-filter 


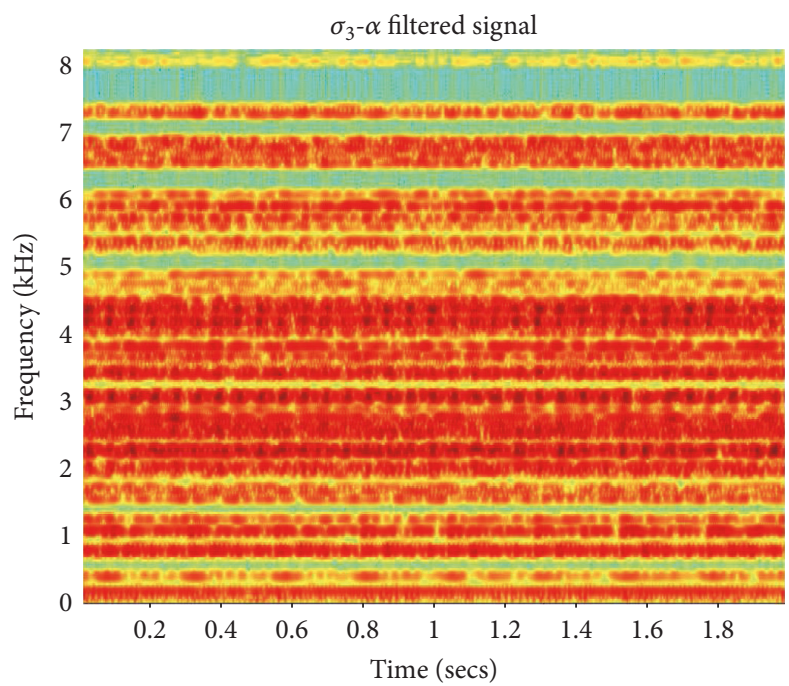

(a)

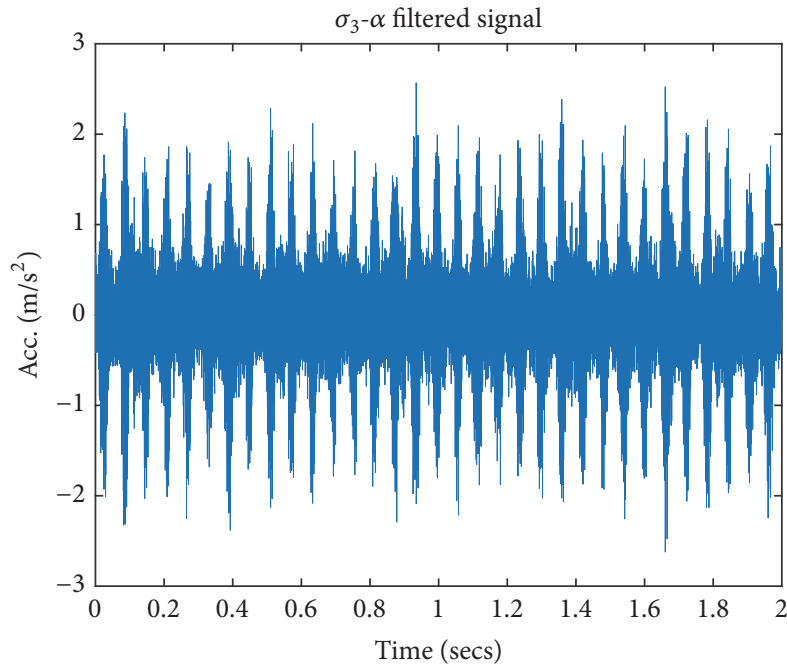

(b)

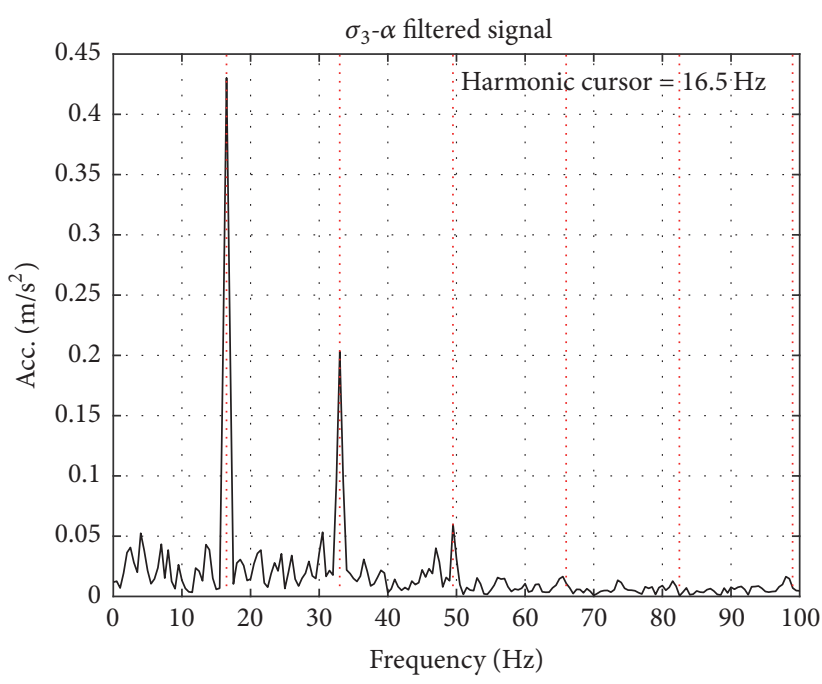

(c)

FIGURE 12: Spectrogram of the signal (a), time waveform of the signal (b), and its envelope spectrum (c).

characteristics which are presented in Figure 9. It can be seen that first three iterations put emphasis on the attenuation of the deterministic component of the signal. Further iterations will result in attenuation of the different components of the signal. In Figure 10 one can see stability parameter value after each filtration. It can be observed that the lowest value of this parameter was estimated for the third filtration. Additionally, we tested iterative procedure for parameter $\alpha$ as SOI enhancement method. However, results after iterative procedure using this parameter were similar to the results after single $\alpha$-filtration.

In Figure 11 one can see results of the iterative filtration using $\sigma$-filter. Procedure has been applied three times resulting in lowest value of stability parameter for the sample. First, one can notice equalization of amplitudes through most of the frequency bands in the spectrogram in Figure 11(a). Furthermore, it can be seen that attenuation of the deterministic components allowed us to obtain frequency of the fault and its harmonic on the envelope spectrum. Detected frequency of the fault is equal to $16.5 \mathrm{~Hz}$. However, still one can not distinct impulsive behavior in the time domain and as such one needs to consider methods allowing for improving SNR. In our case, we have used previously developed $\alpha$-stable distribution stability parameter procedure for enhancement of the IFB.

Combining results obtained using iterative application of $\sigma$-filter with $\alpha$-filter which enhances impulsive components one can observe that result allows one for the detection of the hidden fault. In Figure 12(a) it can be seen that bands containing impulsive component connected to the fault have been strongly emphasized. Observing Figures 12(b) and 12(c) it can be seen that hidden fault is now easily observable in both time and frequency domain.

\section{Conclusions}

In the paper a novel procedure for SOI denoising is proposed. It is $\alpha$-stable distribution based data-driven filters set that 
iteratively attenuates high energy low frequency components by $\sigma$-filtering and finally enhances impulsive components by $\alpha$-filtering. As stoppage criterion for iterative $\sigma$-filtering, stability parameter of the signal in time domain was applied as a measure of impulsivity in the signal. Applying $\alpha$-filtering iteratively does not result in improving signal to noise ratio. Application of the procedure was performed on the real data from the complex mechanical system. Measured data was acquired from the gearbox of the belt conveyor's pulley drive. There were two existing faults in the system, first located at $4.1 \mathrm{~Hz}$ frequency and second at $16.5 \mathrm{~Hz}$ frequency. Due to the second being close multiplicative of the first one it was not possible to determine its existence in any domain. Applying novel procedure it was possible to attenuate certain parts of the signal and enhance visibility of the second fault. In other words, we were able to detect both damage types.

\section{Conflicts of Interest}

The authors declare that there are no conflicts of interest regarding the publication of this paper.

\section{Acknowledgments}

This work is partially (A. Wyłomańska) supported by the Framework Programme for Research and Innovation Horizon 2020 under Grant Agreement no. 636834 (DISIRE, Integrated Process Control based on Distributed In Situ Sensors into Raw Material and Energy Feedstock).

\section{References}

[1] P. D. McFadden and J. D. Smith, "Vibration monitoring of rolling element bearings by the high-frequency resonance technique-a review," Tribology International, vol. 17, no. 1, pp. 3-10, 1984.

[2] R. B. Randall and J. Antoni, "Rolling element bearing diagnostics-a tutorial," Mechanical Systems and Signal Processing, vol. 25, no. 2, pp. 485-520, 2011.

[3] P. D. Samuel and D. J. Pines, "A review of vibration-based techniques for helicopter transmission diagnostics," Journal of Sound and Vibration, vol. 282, no. 1-2, pp. 475-508, 2005.

[4] J. Antoni, F. Bonnardot, A. Raad, and M. El Badaoui, "Cyclostationary modelling of rotating machine vibration signals," Mechanical Systems and Signal Processing, vol. 18, no. 6, pp. 1285-1314, 2004.

[5] R. B. Randall, J. Antoni, and S. Chobsaard, "A comparison of cyclostationary and envelope analysis in the diagnostics of rolling element bearings," in Proceedings of the IEEE Interntional Conference on Acoustics, Speech, and Signal Processing (ICASSP '00), pp. 3882-3885, Istanbul, Turkey, June 2000.

[6] J. Obuchowski, A. Wylomanska, and R. Zimroz, "Stochastic modeling of time series with application to local damage detection in rotating machinery," Key Engineering Materials, vol. 569-570, pp. 441-448, 2013.

[7] J. Obuchowski, A. Wylomanska, and R. Zimroz, "Two-stage data driven filtering for local damage detection in presence of time varying signal to noise ratio," Vibration Engineering and Technology of Machinery, vol. 23, pp. 401-410, 2014.
[8] J. Antoni, "Fast computation of the kurtogram for the detection of transient faults," Mechanical Systems and Signal Processing, vol. 21, no. 1, pp. 108-124, 2007.

[9] T. Barszcz and A. JabŁoński, "A novel method for the optimal band selection for vibration signal demodulation and comparison with the Kurtogram," Mechanical Systems and Signal Processing, vol. 25, no. 1, pp. 431-451, 2011.

[10] J. Antoni and R. B. Randall, "The spectral kurtosis: application to the vibratory surveillance and diagnostics of rotating machines," Mechanical Systems and Signal Processing, vol. 20, no. 2, pp. 308-331, 2006.

[11] G. K. Chaturved and D. W. Thomas, "Adaptive noise cancelling and condition monitoring," Journal of Sound and Vibration, vol. 76, no. 3, pp. 391-405, 1981.

[12] J. Lin, M. J. Zuo, and K. R. Fyfe, "Mechanical fault detection based on the wavelet de-noising technique," Journal of Vibration and Acoustics, vol. 126, no. 1, pp. 9-16, 2004.

[13] S. Braun, "The synchronous (time domain) average revisited," Mechanical Systems and Signal Processing, vol. 25, no. 4, pp. 1087-1102, 2011.

[14] J. Obuchowski, R. Zimroz, and A. Wyłomańska, "Blind equalization using combined skewness-kurtosis criterion for gearbox vibration enhancement," Measurement, vol. 88, pp. 34-44, 2016.

[15] R. B. Randall and N. Sawalhi, "A new method for separating discrete components from a signal," The Sound and Vibration, vol. 45, no. 5, pp. 6-9, 2011.

[16] J. Obuchowski, A. Wyłomańska, and R. Zimroz, "Selection of informative frequency band in local damage detection in rotating machinery," Mechanical Systems and Signal Processing, vol. 48, no. 1-2, pp. 138-152, 2014.

[17] G. Żak, J. Obuchowski, A. Wyłomańska, and R. Zimroz, "Novel $2 \mathrm{D}$ representation of vibration for local damage detection," Mining Science, vol. 21, pp. 105-113, 2014.

[18] R. Makowski and R. Zimroz, "New techniques of local damage detection in machinery based on stochastic modelling using adaptive Schur filter," Applied Acoustics, vol. 77, pp. 130-137, 2014.

[19] Y. Lei, J. Lin, Z. He, and M. J. Zuo, "A review on empirical mode decomposition in fault diagnosis of rotating machinery," Mechanical Systems and Signal Processing, vol. 35, no. 1-2, pp. 108-126, 2013.

[20] Z. Feng, M. Liang, and F. Chu, "Recent advances in timefrequency analysis methods for machinery fault diagnosis: a review with application examples," Mechanical Systems and Signal Processing, vol. 38, no. 1, pp. 165-205, 2013.

[21] Z. K. Peng and F. L. Chu, "Application of the wavelet transform in machine condition monitoring and fault diagnostics: a review with bibliography," Mechanical Systems and Signal Processing, vol. 18, no. 2, pp. 199-221, 2004.

[22] R. Yan, R. X. Gao, and X. Chen, "Wavelets for fault diagnosis of rotary machines: a review with applications," Signal Processing, vol. 96, pp. 1-15, 2013.

[23] M. Shao and C. L. Nikias, Signal Processing with Alpha-Stable Distribution and Applications, John Wiley and Sons Inc, New York, NY, USA, 1995.

[24] G. Żak, A. Wyłomańska, and R. Zimroz, "Data-driven vibration signal filtering procedure based on the alpha-stable distribution," Journal of Vibroengineering, vol. 18, no. 2, pp. 826-837, 2016.

[25] G. Żak, A. Wyłomańska, and R. Zimroz, "Application of alphastable distribution approach for local damage detection in 
rotating machines," Journal of Vibroengineering, vol. 17, no. 6, pp. 2987-3002, 2015.

[26] P. G. Georgiou, "Alpha-stable modeling of noise and robust timedelay estimation in the presence of impulsive noise," IEEE Transactions on Multimedia, vol. 1, no. 3, pp. 291-301, 1999.

[27] G. Yu, C. N. Li, and J. F. Zhang, "A new statistical modeling and detection method for rolling element bearing faults based on alpha-stable distribution," Mechanical Systems and Signal Processing, vol. 41, no. 1-2, pp. 155-175, 2013.

[28] G. Żak, A. Wyłomańska, and R. Zimroz, "Local damage detection methods based on the stochastic modeling techniques," in Proceedings of the 24th Mediterranean Conference on Control and Automation (MED '16), pp. 355-360, Athens, Greece, June 2016.

[29] J. B. Allen, "Short term spectral analysis, synthesis, and modification by discrete Fourier transform," IEEE Transactions on Acoustics, Speech, and Signal Processing, vol. 25, no. 3, pp. 235238, 1977.

[30] G. Samorodnitsky and M. Taqqu, Stable Non-Gaussian Random Variables, Chapman and Hall, London, UK, 1994.

[31] I. A. Koutrouvelis, "Regression-type estimation of the parameters of stable laws," Journal of the American Statistical Association, vol. 75, no. 372, pp. 918-928, 1980. 


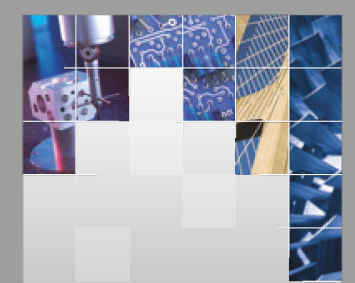

\section{Enfincering}
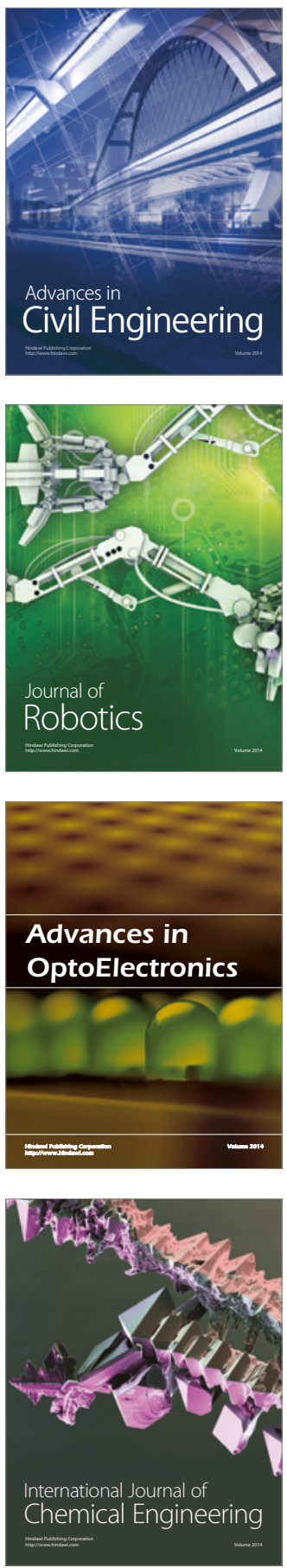

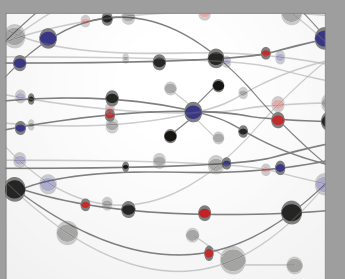

The Scientific World Journal

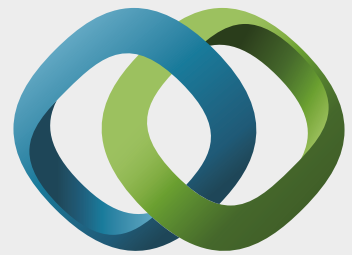

\section{Hindawi}

Submit your manuscripts at

https://www.hindawi.com
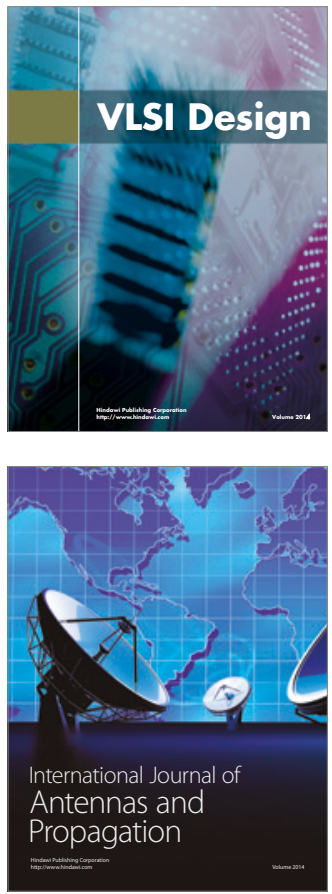

\section{Rotating}

Machinery
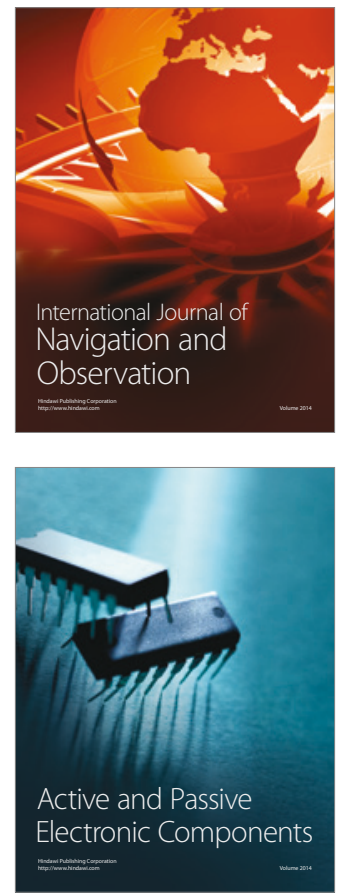
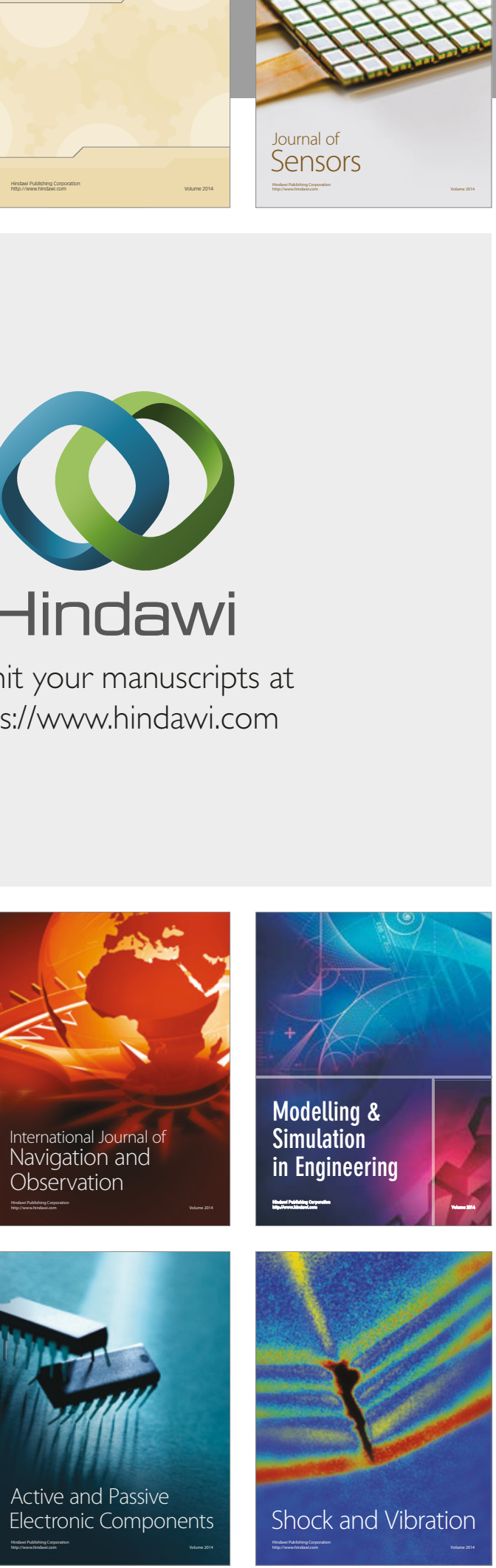
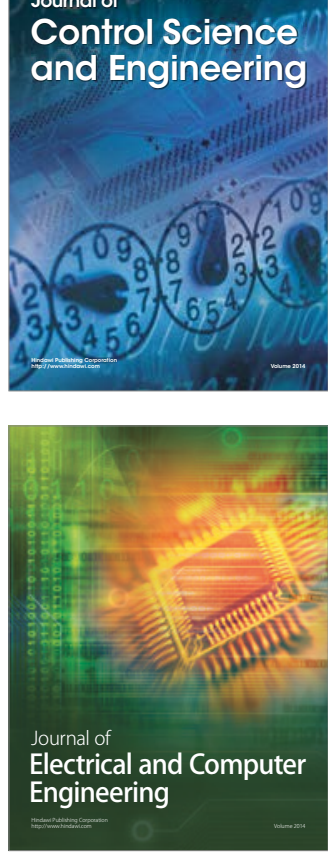

Distributed

Journal of

Control Science

and Engineering
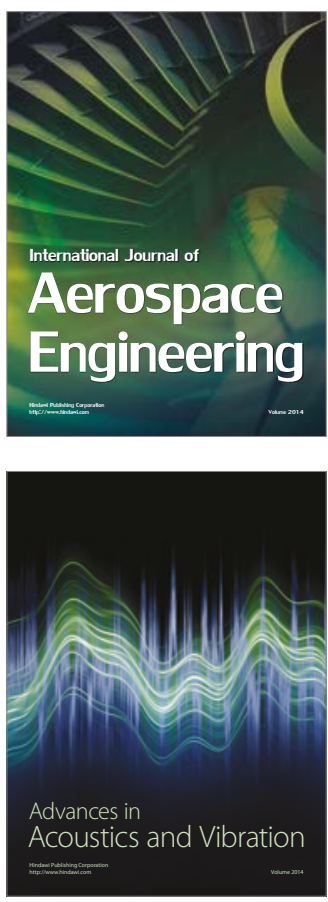

Sensor Networks 